\title{
Discussion on the Application of CG Technology in Stage Art
}

\author{
Yaojie Liu ${ }^{1, *}$ \\ ${ }^{1}$ Ningbo University of Finance \& Economics, Ningbo, Zhejiang, 315175, China \\ ${ }^{*}$ Corresponding author. Email: liuruhua1314@gmail.com
}

\begin{abstract}
In the history of stage art development, technology and art have always been like a shadow. With the rapid development of technology, the combination of art and technology has long become inseparable in the current domestic and international stage art creation trends. Technology has become an important support and carrier for literary creation. The stage art embodied by the combination of projection, sound, light, electricity and other multimedia technologies and virtual reality technology has developed into a powerful cultural industry, which meets people's modern aesthetics and cultural consumption needs. CG is the abbreviation of "computer graphics" in English. It is the general term for computer graphics and image processing technology. It is a combination of modern science and technology and traditional plastic arts.
\end{abstract}

Keywords: CG technology, Stage art, Application

\section{INTRODUCTION}

$\mathrm{CG}$ is a tool used to make visual design and production. CG technology is a combination of computer technology and art and used in the creation of art. With the continuous development of science and technology, CG technology has also been greatly improved. It has a wide range of applications, mainly used in film and television special effects production, architectural modeling design, stage art, and so on. The history of the application of CG technology on the stage is relatively short. Generally, its application is more in the Spring Festival Gala, stage variety show and large-scale concerts, but gradually it has some applications in other stages. CG technology has become an important part of stage art. The application of CG animation technology on the stage can be used throughout the stage.

\section{THE APPLICATION OF CG TECHNOLOGY IN THE PREPARATION PHASE OF STAGE ART}

In the preparation phase of stage art, the main form of CG technology application is to use animation technology to realize a virtual preview before the performance and present a stage environment for a complete performance in advance, which can effectively predict the situation that may occur in the performance, provide the directors with a basis for early observation and late-stage adjustments, and effectively reduce the cost of stage construction and on-site handling of personnel. ${ }^{[1]}$

\section{THE APPLICATION STATUS OF CG TECHNOLOGY IN THE STAGE}

Multimedia, as a technical means, expands the space limitation, realizes the diversification and three-dimensional stage space, and then increases the performance of the performance. The accompanying interactive experience has also become part of the performance, so immersive and mobile performances appear.

\subsection{It Can "Resurrect" Deceased Idols to Perform on the Same Stage}

CG animation technology was also applied in the Asian musical king Jay Chou's Opus Jay 2013 World Tour to sing with the deceased Asian singing queen Teresa Teng. Thanks to the combination of cutting-edge "virtual image reconstruction technology" and traditional projection technology, Teresa Teng walked out from the distant memories of the audience. At Jay Chou's vocal concert in September, in front of the 
carved gates of the "Mortal Life Inn" on the stage of the Little Arena, Teresa Teng, dressed in a white cheongsam, slowly rose from the stage, as elegant as ever. The vivid movements, demeanor and singing made the audience in the audience difficult to distinguish for a while. ${ }^{[2]}$ Two generations of iconic figures in the music industry have completed three cross-time duets on the same stage. Virtual human performance is a pioneering artistic project. Using CG technology to produce a virtual human requires a series of complex procedures and requires a lot of time and energy.

The "resurrection" of Teresa Teng was not only in Jay Chou's vocal concert, but also on the stage of Hunan Satellite TV's "Happy Embrace 2011 Beijing Time" New Year's Eve concert, it also recalled Queen Teresa. The deceased famous singer Teresa Teng "lively" stood and sang with Yisa $\mathrm{Yu}$ on the same stage, communicating with Yisa Yu appropriately, which made hundreds of millions of audiences across the country watch the performance with a sigh. In diva Faye Wong's "Rebirth Concert", the same technology was also applied in a prominent way. Two Faye Wong appeared on the same stage at the same time. This technology allows the audience's familiar deceased star to be "reborn" on the stage. It not only produces a three-dimensional image, but also allows the image to interact with the performer and complete the performance together, thus arousing the audience's emotional identification and viewing resonance, which produces shocking performance effects. ${ }^{[3]}$

\subsection{It Can Realize Co-Acting with the Two-Dimensional Characters on the Same Stage}

On November 1st, 2020, in a stage full of technology and a future scene that is both virtual and real, the five virtual idols of the Infinite King Group used CG technology to achieve an amazing cross-dimensional performance with the real idol Song Qian. In the preparation phase of the stage, images are formed by performing dance moves and music through motion capture, and the live stage drives these images through tracking equipment and engines. Behind the avant-garde stage is virtual idols, a new industry that is highly dependent on technical standards and team operations. They are better managed but more difficult to shape. The behind-the-scenes team needs to continuously create expression scenes for them to interact with the audience, including a lot of effort to figure out how to achieve the highest quality technical output within the controllable range, and how to deliver the correct information to the audience. ${ }^{[4]}$

First of all, in order to pursue rich lens scheduling and the real effect of virtual and real integration, the team used 6-camera tracking equipment and engines to ensure the realization of large panoramas, panoramas, medium and close shots, close-ups, and large-scale motion shots; in particular, the five virtual idols and Song Qian's close-range formation changes, singing and jumping interactions, etc., have undergone multiple rounds of dynamic rehearsal design deliberations in the early stage, and the on-site guidance team accurately presented them in a time code synchronization manner.

Secondly, in the face of complex lighting changes in indoor concerts and stage-like visual effects such as immersive large screens, in order to obtain stable and effective tracking data of Steadicam 360-degree rotating lens and large-range rocker motion lens, and the AR team designed infrared tracking technical solutions for these special lenses to ensure perfect lens presentation.

\subsection{CG Technology Applied in Film and Television}

A CG movie refers to a movie in which all the visual products (scene, characters, objects, special effects, etc.) in the entire movie are composed of computer-generated $\mathrm{CG}$ animations or CG pictures. But its visual effects are completely different from traditional 2D cartoons. Generally, offline rendering is used, which is different from real-time rendering of DEMO and engine movies. When we see Forrest Gump appearing in the same frame as President Nixon in the movie, we will be shocked. This method of grafting historical material films to actors through CG technology was also talked about by later filmmakers. Even so, it is not difficult for us to find that the heroic wars of these great directors in the 1990s are all based on the movie itself and the story of the characters. While creating, the directors carefully used movie special effects based on the idea of "filling the gaps in imagination with $\mathrm{CG}^{\prime},{ }^{[5]}$

In the CG effect of film and television, the exaggerated or distorted expressions in the characters are a kind of abnormal state. Compared with the plain and normal expressions, they can be confused before the audience's critical inspection, and bring the audience a better viewing experience.

\section{THE ADVANTAGES OF CG TECHNOLOGY IN STAGE ART}

Using peripheral communication as the theoretical basis can build a new path for the de The stage image is inseparable from $\mathrm{CG}$, and special effects technology creates $3 \mathrm{D}$ effects. This breakthrough creativity and application has pushed the multimedia stage design and the effects of stage and video to the extreme. And this kind of holographic projection technology, which allows people to easily enjoy the 3D feast effect with the naked eye, is becoming more and more uncommon on the stage, which shows the popularity of 3D technology. Except for Teresa Teng, many deceased stars have left good memories for the audience. We will also feel warm 
when we can use CG technology to retrieve our memories of them. From the time when Audrey Hepburn performed the "Chocolate Temptation" via travelling through time and space, to today's Teresa Teng "returning" to the public vision of ordinary people, the charm of CG technology can be seen. ${ }^{[6]}$

The traditional stage usually changes the stage scene through the switch of scenes, which is more complicated. The CG technology can realize the transformation between different multimedia video contents and realize the narrative ability of the stage story and the switching of time and space. Whether it is from modern to future, modern to ancient times, mountains to plains, or Gobi to snow-capped mountains, a natural transition can be achieved. The use of CG technology to create images in stage art can achieve dynamic effects that are difficult to achieve in traditional stages, which makes the stage more layered, plentiful and changeable, and uses digital multimedia images to give the audience an immersive experience.

The most prosperous period of stage art was in the first 50 years of the last century, after which stage art began to decline gradually. The main reason is that the rise of film and television art makes stage art less attractive to audiences. But stage art has not disappeared, because stage art is performed directly in front of the audience. This form of expression also shows that although stage art is difficult to survive in the era of diversification, it will not really die out. And with the application of CG technology in stage art, it can break the space limitation of traditional stage art, and the current stage art has begun to revive. CG technology uses its own advantages to expand the space of stage art to the greatest extent. This breakthrough in time and space brings a direct impression to the audience through technology. ${ }^{[7]}$

\section{THOUGHTS ON THE APPLICATION OF CG TECHNOLOGY IN STAGE ART}

The application of CG technology in stage art is obviously more successful from the audience's feedback. From the perspective of the performance effect of CG technology in stage art, it is mainly used to create fantastic and changeable stage art effects, and attract the audience's attention by bringing a strong visual impact to the audience. But after all, CG technology is only used to assist stage exhibitions. It is a virtual performance tool. You cannot rely solely on the colorful effects of $\mathrm{CG}$ to attract audiences. That way, you lose the connotation that stage art works really want to realize and perform the performance of stage actors. Weaken, put the cart before the horse. The focus should be placed on the stage works or film and television works themselves, only to polish enough wonderful works, and to choose artistic techniques to enrich the connotation of stage performances, at the same time, it should be connected with the audience's aesthetic needs, combining stage performances with CG effects, and truly showing what the work wants to convey in front of the audience, so as to make the audience really feel the charm of stage art, develop a new form of art expression that is widely accepted by the audience, and realize the integration of art and technology. ${ }^{[8]}$

Stage art is a second creation based on script literature, which contains many elements such as director's creativity, actor performance, and props and scenery. When using CG technology, it is necessary to focus on the ideological connotation of the script, starting from the needs of the performance of stage art, and aiming at the complete display of stage art. It cannot be mechanically made and deviate from the theme of stage art. The application of CG technology in stage art is to integrate technology and art to better show the connotation of stage art, rather than blindly show off skills and ignore the essence of stage performance.

CG technology can not only increase the effect of stage art in the stage art, but also can be used in the stage of the drama. The introduction of it into the traditional opera stage and other non-heritage material protection stages is of great significance for their inheritance. The advantages of CG technology are used to record performances, learn and appreciate through holographic technology, and preserve these intangible heritages completely and accurately through $\mathrm{CG}$, and finally put these intangible heritages on the art stage to reproduce the style of the year.

When the times continue to develop, CG technology is integrated into stage art, opening up a new world for the future development of stage art, and can better serve stage art and make an important contribution to the revival of stage art.

\section{CONCLUSION}

This paper mainly to discuss the application of $\mathrm{CG}$ technology in stage art. This paper with the application of CG technology in the early stage of stage art. CG technology can achieve preview effects in stage art. Secondly, the status quo of the application of $\mathrm{CG}$ technology in the stage began to be analyzed. At present, the use of CG technology to achieve good stage effects is Jay Chou's dream of co-acting with Teresa Teng in the concert, realizing co-acting with the two-dimensional characters and so on. CG technology breaks the space and time constraints of traditional stage art, brings a brand-new visual feast to the audience, and at the same time brings a brand-new aesthetic concept to the audience, which is in line with the development trend of contemporary stage art. Finally, through thinking about the application of CG technology to stage art, it is understood that no matter how far the CG technology develops, As the main factor in stage art, people are not 
only the source of creation but also the performer of art. The connotation of art is conveyed to the audience through stage actors, directors and staff behind the scenes. The true connotation of stage art cannot be conveyed only through the operation of cold machines.

\section{REFERENCES}

[1] Li Li, Discussion on the application form and thinking of multimedia technology in stage art, in: Entertainment Technology, vol. 2020(10), China Entertainment Technology Association, Beijing, 2020(10), pp.42-44. DOI: 10.3969/j.issn.1674-8239.2020.10.010.

[2] Jun Sun, Analysis of the application of holographic projection technology in the stage art of Yue opera, in: China Newspaper Industry, vol. 2020(20), China Newspaper Association, Beijing, 2020(20), pp. 68-69. DOI: not available at present.

[3] Lili Zhao, Innovation and application of stage technology in the creation of drama and stage art, in: Public Communication of Science \& Technology, vol. 12(17), Chinese Society for Science and Technology Journalism, Being, 2020, 12(17), pp. 123-124. DOI: 10.3969/j.issn.1674-6708.2020.17.058.

[4] Lihua Luo, Overview of the application research of CG technology in stage art, in: Cultural Monthly, vol. 2019(11), China Cultural Media Group Co., Ltd., Beijing, 2019(11), pp. 122-123. DOI: cnki:sun:whyk.0.2019-11-056.

[5] Jiawei Sun, Application of Computer Technology in Drama and Stage Art, in: Oriental Collection, vol. 2018(02), Shanghai Pictorial Publishing House, Shanghai, 2018(02), pp. 99. DOI: not available at present.

[6] Xianmei Ding, Anjian Zhang, Rongting Zhou, Research on the application of augmented reality technology in the design of opera stage art, in: Popular Literature, vol. 2016(18), Hebei Mass Art Centre, Shijiazhuang, 2016(18), pp. 262-263. DOI: 10.3969/j.issn.1007-5828.2016.18.223.

[7] Yili Yan, Analysis of the application of holographic technology in theatrical stage art, in: Science and Technology of West China, vol. 12(11), Chinese Academy of Sciences, Beijing, 2013, 12(11), pp. $47+53$.

DOI: 10.3969/j.issn.1671-6396.2013.11.020.

[8] Xin Du, On the application of multimedia technology in stage art, in: Popular Literature, vol. 2013(06), Hebei Mass Art Centre, Shijiazhuang, 2013(06), pp. 278-279. DOI: 10.3969/j.issn.1007-5828.2013.06.220. 